\title{
Detection and control of combustion instability based on the concept of dynamical system theory
}

\author{
Hiroshi Gotoda, ${ }^{*}$ Yuta Shinoda, Masaki Kobayashi, and Yuta Okuno \\ Department of Mechanical Engineering, Ritsumeikan University, 1-1-1 Nojihigashi, Kusatsu-shi, Shiga 525-8577, Japan \\ Shigeru Tachibana ${ }^{\dagger}$ \\ Propulsion Systems Research Group, Institute of Aeronautical Technology, Japan Aerospace Exploration Agency, 7-44-1 Jindaiji-Higashii, \\ Chofu, Tokyo 182-8522, Japan
}

(Received 25 July 2013; published 11 February 2014; corrected 5 March 2014)

\begin{abstract}
We propose an online method of detecting combustion instability based on the concept of dynamical system theory, including the characterization of the dynamic behavior of combustion instability. As an important case study relevant to combustion instability encountered in fundamental and practical combustion systems, we deal with the combustion dynamics close to lean blowout (LBO) in a premixed gas-turbine model combustor. The relatively regular pressure fluctuations generated by thermoacoustic oscillations transit to low-dimensional intermittent chaos owing to the intermittent appearance of burst with decreasing equivalence ratio. The translation error, which is characterized by quantifying the degree of parallelism of trajectories in the phase space, can be used as a control variable to prevent LBO.
\end{abstract}

DOI: 10.1103/PhysRevE.89.022910

PACS number(s): 05.45.Tp, 47.70.Pq

\section{INTRODUCTION}

Self-excited oscillations caused by closed-loop coupling between unsteady heat-release fluctuations, acoustic velocity, and/or the pressure field are an important thermoacoustic instability of much interest in wide a spectrum of combustion, thermal fluid, and physics fields [1-10]. The development of new engine systems using the concept of thermoacoustic instability has recently been attempted by Biwa and coauthors in the field of physics [7-10]. On the contrary, the complex interaction between the combustion process and the acoustic field inside combustors generates thermoacoustic combustion oscillations with nonlinear dynamic behavior, leading to serious damage in practical combustors such as propulsion and land-based gas-turbine engines. There have been many experimental and numerical investigations exploring the physical mechanisms responsible for the excitation of thermoacoustic combustion oscillations regardless of the existence or nonexistence of a swirling flow [11-17]. As a representative study on the flame-acoustic interaction mechanisms in a swirl-stabilized premixed combustor, Polifke and Lawn [15] have proposed three essential mechanisms by which acoustic velocity fluctuations give rise to perturbations in equivalence ratio, flame front kinematics, turbulence intensity, and length scale, possibly including large-scale coherent vortex structures. All these perturbations result in heat-release fluctuations. More recently, the physical mechanisms identified to date in the field of combustion physics have been summarized in a review paper [18] and book [19].

Lean premixed combustion is a highly promising method for effectively reducing nitrogen oxide $\left(\mathrm{NO}_{\mathrm{x}}\right)$ emission from gas-turbine engines without sacrificing combustion efficiency. However, it is highly susceptible to combustion instabilities such as thermoacoustic combustion oscillations, lean blowout (LBO), and flame flashback. Recently, we showed that with

\footnotetext{
*gotoda@se.ritsumei.ac.jp

${ }^{\dagger}$ tachibana.shigeru@jaxa.jp
}

increasing equivalence ratio, the dynamic behavior of combustion instabilities in a premixed gas-turbine model combustor undergoes a significant transition from fractional Brownian motion in close proximity to LBO to periodic oscillations generated by thermoacoustic combustion oscillations through chaos [20,21]. In addition to thermoacoustic combustion oscillations, LBO is another problem that must be avoided when operating gas-turbine engines, since it leads to severe safety problems and high operational costs. Current gasturbine combustor engines are operated within a wide safety margin far from actual blowout limits due to the difficulty in detecting LBO precursors in engines. Most approaches to detecting or correlating LBO limits are based on the idea that LBO is caused by the competition between characteristic time scales of flow residence and chemical kinetics. For instance, Zhang et al. [22] concluded that the global Damköhler number (given by the ratio of the flow residence and chemical kinetic time scales) describes the onset condition of local flame extinction near LBO, which should have a direct relation with global LBO. The phenomenological approach in their study provides an in-depth understanding of the complex LBO phenomenon and should ultimately lead to the establishment of a design parameter to determine actual LBO conditions. Meanwhile, a novel method of detecting LBO precursors by monitoring signals in engines is of importance for controlling the complex combustion state under low equivalence ratios.

Nonlinear time series analysis based on dynamical system theory conveys striking and revealing information for clarifying the dynamic properties of irregular time series data obtained in a wide range of experiments in the fields of medicine, biology, electronics, mechanical engineering, and many other sciences [23]. It widely covers areas ranging from the quantification of many important invariants such as fractal dimension, Lyapunov exponents, and entropies, which yield a physical description of the dynamical structure, to practical applications such as nonlinear prediction and noise reduction. In fact, it has recently been used to investigate the temporal behavior of combustion and flame front instabilities observed 
in experiments (e.g., a thermal pulse combustor [24], a ducted premixed combustor [3-5], a spark ignition engine [25,26], and swirling premixed flames [27]). These previous works have been devoted to the characterization of complex combustion dynamics from the viewpoint of the self-similar structure and orbital instability in a phase space using the correlation dimension obtained by the Grassberger-Procaccia (GP) algorithm [28], multifractal analysis, and the Lyapunov exponent. Some of the authors have also used more sophisticated nonlinear time series analysis to extract the short-term predictability and longterm unpredictability characteristic of chaos in combustion [20] and flame front instabilities [29]. The importance of our approach has also been discussed from a practical point of view [20,29]. The relevance of chaos to combustion instabilities has thus far been shown by nonlinear time series analysis $[4,5,20,24,29,30]$, but there have been no attempts to propose an online method of detecting an unstable combustion state to appropriately control a combustion system, focusing on the concept of orbital instability in the phase space. Developing a sophisticated method of detecting a precursor of LBO based on the extracted nonlinear nature of the complex dynamics underlying combustion instability is a major challenge in contemporary time series analysis, which will be of interest in nonlinear physics and engineering disciplines.

The main purpose of this study is to propose an online method of detecting combustion instability based on the concept of dynamical system theory. As an important case study relevant to combustion instability encountered in a fundamental and practical combustion system, we deal with combustion dynamics in a laboratory-scale lean premixed gas-turbine model combustor with an axial swirler flame holder. A simple and useful nonlinear time series analysis based on orbital instability in the phase space, referred to as the translation error method [31], represents the degree of parallelism of trajectories in the phase space which has been used to successfully characterize the dynamic properties of different types of flame front instability [27]. In this study, we discuss the applicability of the translation error as an online detector. The translation error is estimated for trajectories in the phase space constructed from short time series data of combustion-chamber pressure fluctuations. On the basis of the extracted nonlinear nature of the unstable combustion state close to LBO, we attempt to control the combustion state so as to prevent LBO using the translation error as a control variable. The intermittent phenomenon of irregular alternation between regular fluctuations and burst is of much interest in the fields of modern nonlinear science and physics. As will be presented in this work, in a narrow range of the equivalence ratio, an interesting intermittent phenomenon is formed, with its pressure fluctuations switching back and forth between regular fluctuations generated by thermoacoustic instability and irregular fluctuations with small amplitude. We therefore investigate the dynamic behavior of this intermittent phenomenon from the viewpoint of dynamical system theory, in addition to proposing an online method of detecting combustion instability. This paper is organized as follows. In Sec. II, we briefly describe the experimental apparatus and the detection method of combustion instability. In Sec. III, we present the results and discussion, and our conclusions are given in Sec. IV.

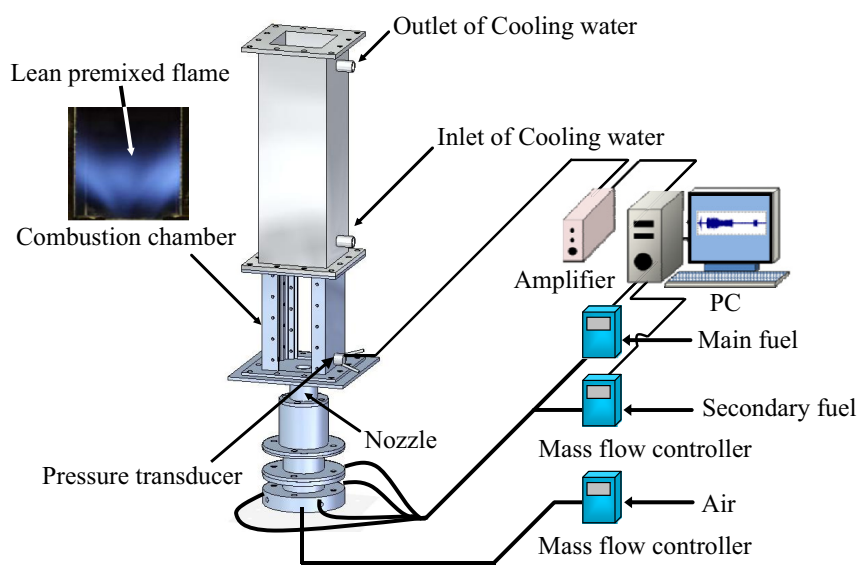

FIG. 1. (Color online) Experimental system.

\section{EXPERIMENTAL APPARATUS AND DETECTION METHOD OF COMBUSTION INSTABILITY}

The configuration of our experimental system is shown in Fig. 1. The combustion test rig is composed of mass flow controllers, a nozzle, an axial swirler, and a combustion chamber. The chamber has a length of $620 \mathrm{~mm}$ with a $100 \times$ $100 \mathrm{~mm}^{2}$ cross section. The rest of the chamber is composed of a water-cooled stainless-steel duct. Methane gas is used as the main fuel. There is a secondary fuel line for modifying the total fuel flow rate near the LBO. An axial swirler, which is normally adopted for holding flame in most practical combustion devices, is installed at the inlet of the combustion chamber. The inlet air temperature and volume flow rate (mean axial flow velocity at the inlet of the combustion chamber) are $300 \mathrm{~K}$ and $148 \mathrm{~L} / \mathrm{min}(5.8 \mathrm{~m} / \mathrm{s})$, respectively. The equivalence ratio $\phi$ is decreased from 0.74 to 0.46 , because it is under these conditions that the combustion instability changes from thermoacoustic combustion oscillations to LBO. The pressure fluctuations $p^{\prime}$ are measured by a pressure transducer (JTEKT Products, PD104K-10 kPa) as a physical quantity that represents the nonlinear dynamics of the combustion state. In practice, a pressure transducer is commonly preferable for monitoring the combustor status in practical combustion systems from the viewpoint of robustness. The pressure transducer is located on the wall of the combustion chamber, $20 \mathrm{~mm}$ from its inlet. Signals from the pressure transducer are acquired through a data acquisition system (LabVIEW system, National Instruments). The sampling frequency of $p^{\prime}$ is $5 \mathrm{kHz}$. The noise level of the pressure fluctuation measurement is within $\pm 0.004 \mathrm{kPa}$ in the absence of a flow, which is small compared with the pressure fluctuations in combustion instabilities we observed in this work.

If the determinism is clearly visible in a time series obtained in experiments, the neighboring trajectories in the phase space will have similar directions. However, as the determinism is lost, the trajectories diverge with time due to the orbital instability in the phase space. On the basis of this concept, we introduce a useful algorithm [31] to extract the determinism from complex time series data obtained in experiments. A phase space based on Takens' embedding theorem [32] is first constructed from $p^{\prime}$. The time-delayed coordinates used for 
the construction of the phase space are expressed as

$$
\mathbf{p}^{\prime}\left(t_{i}\right)=\left[p^{\prime}\left(t_{i}\right), p^{\prime}\left(t_{i}+\tau\right), p^{\prime}\left(t_{i}+2 \tau\right), \ldots, p^{\prime}\left(t_{i}+(D-1) \tau\right)\right],
$$

where $i=0,1, \ldots, n$ ( $n$ is the data number of the time series), $\mathbf{p}^{\prime}\left(t_{i}\right)$ are the phase space vectors, $p^{\prime}\left(t_{i}\right)$ are the pressure fluctuations at time $t_{i}, D$ is the embedding dimension, that is, the dimension of the phase space, and $\tau$ is the time lag. In this work, $D$ is set to 3 . As reported in previous studies [20,29], an appropriate choice for the time lag is made using mutual information. After randomly selecting a vector $\mathbf{p}^{\prime}\left(t_{i}\right)$ from the phase space, we find its $K$ nearest neighbors $\mathbf{p}^{\prime}\left(t_{k}\right)$ with $k$ from 1 to $K$. Here, $K$ is the number of neighboring points to $\mathbf{p}^{\prime}\left(t_{i}\right)$. By setting the images of all the vectors to $\mathbf{p}^{\prime}\left(t_{k}+T \Delta t\right)$ at a suitable time interval $T$, the diversity in the directions of neighboring trajectories is measured in terms of the translation error $E_{\text {trans }}$, defined by

$$
\begin{aligned}
E_{\text {trans }} & =\frac{1}{K+1} \sum_{k=0}^{K} \frac{\left\|\mathbf{v}\left(t_{k}\right)-\overline{\mathbf{v}}\right\|^{2}}{\|\overline{\mathbf{v}}\|^{2}}, \\
\overline{\mathbf{v}} & =\frac{1}{K+1} \sum_{k=0}^{K} \mathbf{v}\left(t_{k}\right) .
\end{aligned}
$$

Here, $\mathbf{v}\left(t_{k}\right)\left[=\mathbf{p}^{\prime}\left(t_{k}+T \Delta t\right)-\mathbf{p}^{\prime}\left(t_{k}\right)\right]$ approximates the tangential vectors of trajectories at time $t_{k} . T$ and $K$ are set to 8 and 5 , respectively, for the estimation of $E_{\text {trans. }}$. The more parallel the trajectories are to each other, that is, the more visible determinism there is in the time series, the closer $E_{\text {trans }}$ will be to zero. In the estimation of the translation error, the stochastic error can be reduced by obtaining the medians of $E_{\text {trans }}$ for $Q$ sets of $M$ randomly chosen $\mathbf{p}^{\prime}\left(t_{i}\right)$ and then taking the mean of the $Q$ medians. In a preliminary test, we systematically varied $M$ and $Q$ from 50 to 500 and from 1 to 10, respectively. The values of $M$ and $Q$ within these ranges were found to have little effect on the translation error. On the basis of the preliminary test, $M$ and $Q$ are set to 100 and 1 in this work, respectively. $E_{\text {trans }}$ is calculated for the trajectories of the phase space constructed from short time series data of $p^{\prime}$. In this work, $E_{\text {trans }}$ is obtained every $100 \mathrm{~ms}$, that is, the library data of the phase space consists of $100 \mathrm{~ms}$ time series data of $p^{\prime}$.

\section{RESULTS AND DISCUSSION}

Time variations of the pressure fluctuations $p^{\prime}$, threedimensional phase space $\left[p^{\prime}(t), p^{\prime}(t+\tau), p^{\prime}(t+2 \tau)\right]$, and translation error $E_{\text {trans }}$ for $\phi=0.62$ are shown in Fig. 2. Irregular pressure fluctuations with small amplitudes suddenly appear in the regular fluctuations with large amplitudes generated by thermoacoustic combustion oscillations. The trajectories at $t=5.0-5.2 \mathrm{~s}$ fill the core of the phase space and do not appear to exhibit a strong deterministic nature, while they become limit cycles with a large width at 15.0-15.2 s. Kabiraj and Sujith [33] recently found that intermittent bursts with large amplitudes irregularly appear in periodic oscillations with small amplitudes in a ducted laminar premixed conical flame. They showed the presence of type-II intermittency using a recurrence analysis based on dynamical system theory. On the basis of our previous studies [20,21], the entire flow

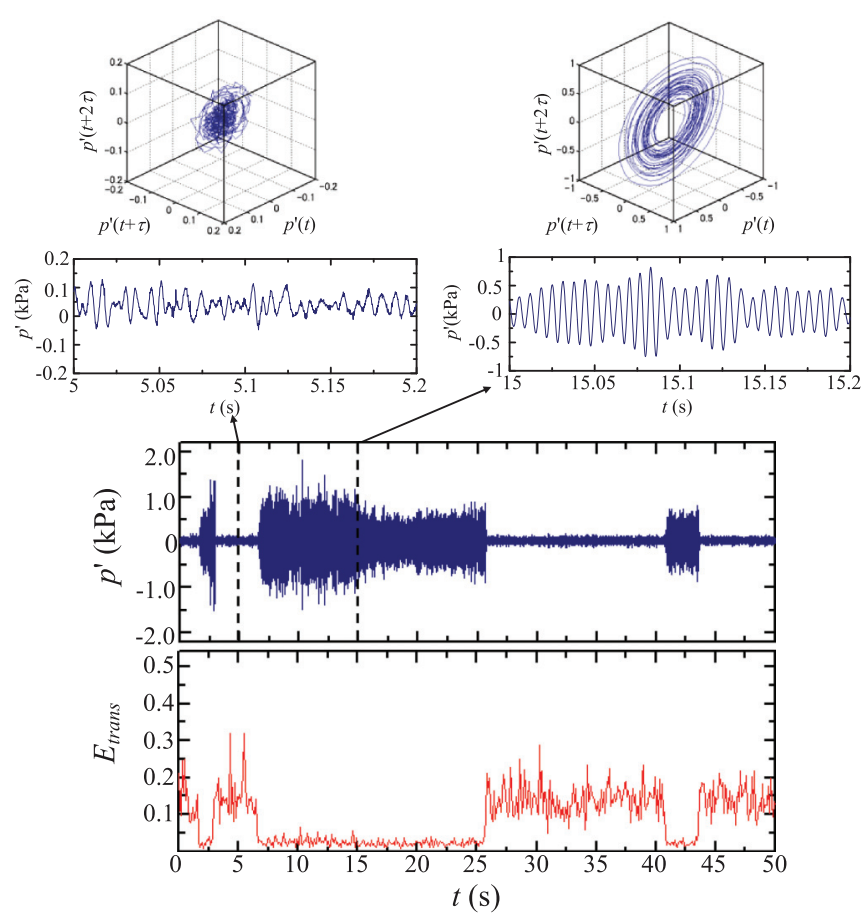

FIG. 2. (Color online) Time variations of pressure fluctuations $p^{\prime}$, three-dimensional phase space $\left(p^{\prime}(t), p^{\prime}(t+\tau), p^{\prime}(t+2 \tau)\right)$, and translation error $E_{\text {trans }}$ for equivalence ratio $\phi=0.62$. The temperature of the inlet air is $300 \mathrm{~K}$. The volume flow rate of the inlet air is $148 \mathrm{~L} / \mathrm{min}$. The amplitude of $p^{\prime}$ drastically changes with the onset of intermittency. $E_{\text {trans }}$ responds to the drastic change in $p^{\prime}$.

field inside our current combustion chamber predominantly consists of two large-scale recirculation regions: one is a vortex breakdown bubble in the wake of the center body due to swirling flow, and the other is an outer recirculation region located in the dump plate due to the backward-facing step flow. In the absence of thermoacoustic combustion oscillations, the entire flame is sustained by the vortex breakdown bubble and stands off from the centerbody. When thermoacoustic combustion oscillations are induced, the flame base propagates into the upstream flow and becomes attached to the axial swirl vane. The entire flame exposed to the thermoacoustic combustion oscillations is sustained by both recirculation regions, with the roll up of the flame caused by vortical structures probably stemming from convective interaction in the shear layers. Although the overall dynamic behavior of the pressure fluctuations with intermittency observed in this work differs from that in a ducted laminar premixed conical flame [33], on the basis of our observation, it is conceivable that the abrupt switching between these states results in the formation of the intermittent phenomena. The implementation of laser diagnostics, such as OH-PLIF and PIV measurements [34], is required in a future work to obtain a more comprehensive understanding of the dynamics of the thermoacoustic combustion oscillations observed in our current experimental system. As shown in Fig. 2, when the amplitude of $p^{\prime}$ drastically changes with the onset of intermittency, $E_{\text {trans }}$ also changes simultaneously. The response of the translation error to the drastic change in pressure fluctuations indicates that it is feasible to use the translation error to detect a drastic change in 
the combustion state in the lean premixed gas-turbine model combustor. In this study, we investigate the dynamic behavior of the intermittent pressure fluctuations using the recurrence plots and a sophisticated nonlinear forecasting method based on orbital instability in the phase space. Note that there have been no attempts in previous studies on combustion to discuss the possible existence of chaos in the intermittent pressure fluctuations from the viewpoint of nonlinear forecasting.

Recurrence plots (RPs), introduced by Eckmann et al. [35], are two-dimensional representations suitable for qualitatively extracting hidden order and disorder pattern structures in a phase space, and have been applied to a wide range of experimental data sets in chemistry and physics. In RPs, black and white dots are commonly used to express values of one and zero, respectively, from the binary matrix $\mathbf{R}_{i, j}\left[=\Theta\left(\varepsilon-\left\|\mathbf{p}^{\prime}\left(t_{i}\right)-\mathbf{p}^{\prime}\left(t_{j}\right)\right\|\right), i, j=1, \ldots, N\right.$, where is $\Theta$ the Heaviside function and $\varepsilon$ is a threshold parameter]. In this work, we use the color-coded version of RPs consisting of 256 gradations without the need to consider the threshold of $\varepsilon$, following Eq. (4):

$$
\mathbf{R}_{i, j}=\frac{\left\|\mathbf{p}^{\prime}\left(t_{i}\right)-\mathbf{p}^{\prime}\left(t_{j}\right)\right\|-\left\|\mathbf{p}^{\prime}\left(t_{i}\right)-\mathbf{p}^{\prime}\left(t_{j}\right)\right\|_{\min }}{\left\|\mathbf{p}^{\prime}\left(t_{i}\right)-\mathbf{p}^{\prime}\left(t_{j}\right)\right\|_{\max }-\left\|\mathbf{p}^{\prime}\left(t_{i}\right)-\mathbf{p}^{\prime}\left(t_{j}\right)\right\|_{\min }} .
$$

Here, a color code at location $(i, j)$ represents the distance between the pair of $\mathbf{p}^{\prime}\left(t_{i}\right)$ and $\mathbf{p}^{\prime}\left(t_{j}\right)$ in the phase space, and various color codes are assigned to different distances. As the deterministic nature appears in the time series data, regularly arrayed structures come to be contained in the RPs. RPs composed of $50,000 \times 50,000$ points, corresponding to $p^{\prime}$ for $0 \leqslant t \leqslant 10 \mathrm{~s}$ at $\phi=0.62$, are depicted in Fig. 3. The pattern corresponding to a burst with small amplitudes is shown in the lower-left corner and center regions. Regularly arrayed geometrical structures do not seem to be contained in these regions. In contrast, they appear in the upper-right corner, corresponding to regularly fluctuating pressures with large oscillation amplitudes, but interestingly, the local structures lose their regularity. Note that the kite-like structure, which is as an important indicator of the presence of type-II intermittency and was found in a ducted laminar premixed combustor [33], is not observed in our experimental system. It is also not observed in our preliminary test using usual RPs consisting of a binary matrix. The results obtained by the RPs indicate the coexistence of large square, rectangular, regular and locally irregular patterns, suggesting the possible existence of chaos.

One of the authors has recently reported that a nonlinear forecasting method taking into account the updating of library data is feasible for predicting the short-term complex temporal behavior of flame front instability [29] and the intermittent chaos in Rayleigh-Bénard convection subjected to a magnetic field [36] from the viewpoint of practical application. The author has also proposed a nonlinear forecasting methodology to capture the chaotic dynamics in spatially extended systems [37]. An important point to note for this methodology is that the dynamic behavior has the short-term predictability and long-term unpredictability characteristic of chaos if the correlation coefficient between the actual and predicted time series data, including the case of the predicted increments



FIG. 3. (Color online) Recurrence plots (RPs) composed of $50,000 \times 50,000$ points for equivalence ratio $\phi=0.62$. The experimental conditions of the inlet air flow are the same as those in Fig. 2. RPs consist of large square, rectangular, regular, and locally irregular patterns, indicating the possible existence of chaos.

of the time series data, gradually decays upon increasing the duration of the actual time series data added to library data. In this methodology, the time series of $p^{\prime}$ at $\phi=0.62$ is initially divided into two parts: the library data and the reference data for comparison with the time series data predicted using the library data. The neighboring vectors in the phase space, denoted by $\mathbf{p}^{\prime}\left(t_{k}\right)(k=1,2, \ldots, K)$, to vector $\mathbf{p}^{\prime}\left(t_{p}\right)$ are searched for among all the points in the phase space constructed from the library data, where $\mathbf{p}^{\prime}\left(t_{p}\right)$ is the final point of the trajectory in the phase space. Note that $\mathbf{p}^{\prime}\left(t_{i}\right)=\left[p^{\prime}\left(t_{i}\right), p^{\prime}\left(t_{i}-\tau\right), p^{\prime}\left(t_{i}-\right.\right.$ $\left.2 \tau), \ldots, p^{\prime}\left(t_{i}-(D-1) \tau\right)\right]$ when applying our proposed nonlinear forecasting method. $p^{\prime}\left(t_{k}+T_{s} \Delta t\right)$ represents the future value corresponding to $\mathbf{p}^{\prime}\left(t_{k}\right)$ after $T_{s}$ time steps. Then, the predicted value $\hat{p}^{\prime}\left(t_{p}+T_{s} \Delta t\right)$ corresponding to $\mathbf{p}^{\prime}\left(t_{p}\right)$ after $T_{s}$ time steps is obtained by the nonlinearity weighted sum of the library data $p^{\prime}\left(t_{k}+T_{s} \Delta t\right)$ shown in Eq. (5):

$$
\hat{p}^{\prime}\left(t_{p}+T_{s} \Delta t\right)=\frac{\sum_{k=1}^{K} \exp \left(-d_{k}\right) \cdot p^{\prime}\left(t_{k}+T_{s} \Delta t\right)}{\sum_{k=1}^{K} \exp \left(-d_{k}\right)},
$$

where $d_{k}=\left\|\mathbf{p}^{\prime}\left(t_{p}\right)-\mathbf{p}^{\prime}\left(t_{k}\right)\right\|$, and $K=1000$ for the nonlinear forecasting. When predicting the time evolution of $p^{\prime}$, the library data is updated so as to continue capturing 
the determinism governing the current temporal behavior, keeping the size of the updated library data constant. In this work, values of $p^{\prime}$ of up to $t=25 \mathrm{~s}$ are used as the library data and those after $t=20 \mathrm{~s}$ are used as the reference.

The correlation coefficient $C$ between the actual and predicted dynamic behaviors in the pressure fluctuations $p^{\prime}$ at $\phi=0.62$ is shown in Fig. 4 as a function of the duration $t_{P}$ of the actual pressure fluctuations added to the library data. $C$ at $t_{P}=2 \times 10^{-4} \mathrm{~s}$ is nearly unity, which means that one-step-ahead prediction of the intermittent pressure fluctuations is feasible with high accuracy. $C$ remains nearly unchanged up to $t_{P}=3.6 \times 10^{-3} \mathrm{~s}$. The appearance of the constant region up to $t_{P}=3.6 \times 10^{-3} \mathrm{~s}$ is the manifestation of the short-term predictability characteristic of chaos. As $t_{P}$ exceeds this critical value, $C$ gradually decreases due to the long-term unpredictability characteristic of chaos associated with intermittently occurring burst regions in the pressure fluctuations. As reported in recent study on a spatially extended system [37], a similar distribution of $C$ in terms of $t_{P}$ for the incremental process should be observed when the dynamical behavior of time series data is chaos. In fact, as shown in Fig. 4, a similar trend is observed for increments

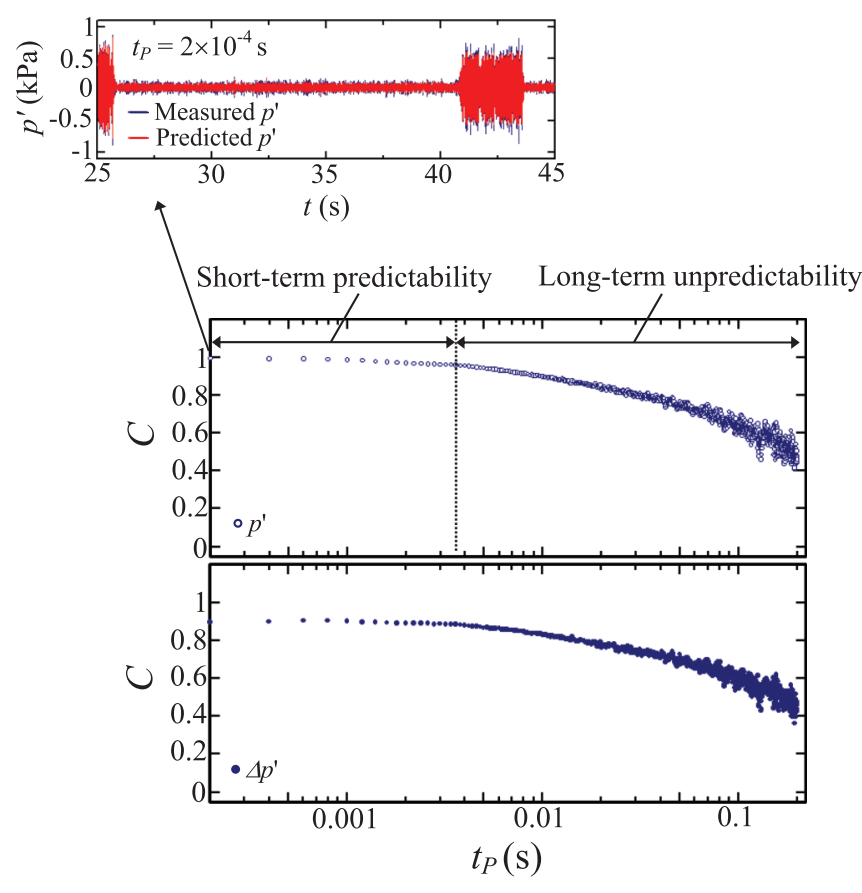

FIG. 4. (Color online) Changes in the correlation coefficient $C$ between the actual and predicted dynamic behaviors in the intermittent pressure fluctuations $p^{\prime}$ at $\phi=0.62$ as a function of the duration $t_{P}$ of the actual pressure fluctuations added to the library data. Changes in $C$ for increments of $\Delta p^{\prime}\left(=p^{\prime}\left(t_{i+1}\right)-p^{\prime}\left(t_{i}\right)\right)$ are also shown as a function of $t_{P}$. The experimental conditions of the inlet air flow are the same as those in Fig. 2. $C$ for $p^{\prime}$ at $t_{P}=2 \times 10^{-4} \mathrm{~s}$ is nearly unity, and remains nearly unchanged up to $t_{P}=3.6 \times 10^{-3} \mathrm{~s}$, which is the manifestation of the short-term predictability characteristic of chaos. The long-term unpredictability characteristic of chaos appears as $t_{P}$ exceeds this critical value. A similar trend is observed for increments of $\Delta p^{\prime}$, indicating the presence of chaotic dynamics. of $\Delta p^{\prime}\left(=p^{\prime}\left(t_{i+1}\right)-p^{\prime}\left(t_{i}\right)\right)$, indicating the presence of chaotic dynamics. In our preliminary test, we obtained a similar trend for the changes in $C$ in terms of $t_{P}$ for periodic fluctuations with an intermittently formed small burst [36], i.e., low-dimensional intermittent chaos produced by Lorenz equations, including the case of incremental process of Lorenz equations solution. This means at least that the dynamic behavior of the intermittent pressure fluctuations at $\phi=0.62$ has the same degree of short-term predictability and long-term unpredictability as the low-dimensional intermittent chaos. As mentioned in our previous work [20], the Lorenz equations in themselves are not a suitable model for dealing with combustion instability, but they enable us to discuss the degree of complexity in the dynamic behavior. On the basis of the results in Figs. 2-4, it appears that the irregular components of the pressure fluctuations with small amplitude affect the relatively regular fluctuations due to thermoacoustic combustion oscillations, resulting in low-dimensional chaotic behavior. Our experimental system differs from the ducted laminar premixed combustor, but the appearance of low-dimensional chaos provides us with significant understanding of the transient process from thermoacoustic combustion oscillations to lean blowout in a laboratory-scale, lean, premixed gas-turbine model combustor with an axial swirler flame holder. Revealing the intermittent characteristics by statistical analysis [26,38] may also be of importance to obtain a better understanding of combustion dynamics in our next study.

The standard deviation of pressure fluctuations $p_{\mathrm{rms}}^{\prime}$ and the translation error $E_{\text {trans }}$ are shown in Fig. 5 as functions of equivalence ratio $\phi$. Note that, similarly to in our previous work [20], in which a different type of gas-turbine model

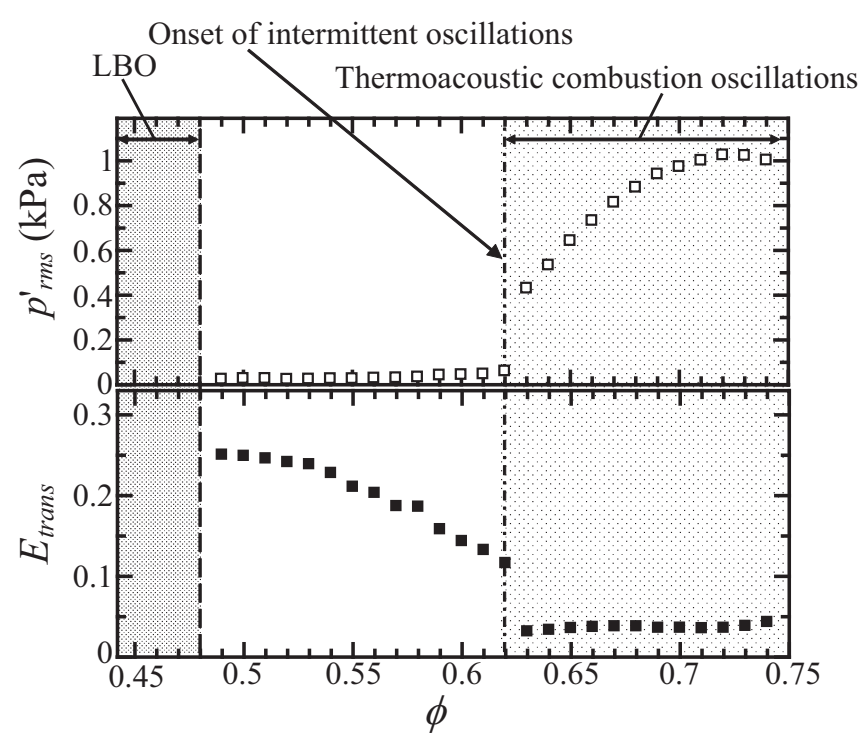

FIG. 5. Standard deviation of pressure fluctuations $p_{\text {rms }}^{\prime}$ and translation error $E_{\text {trans }}$ as functions of equivalence ratio $\phi$. The experimental conditions of the inlet air flow are the same as those in Fig. 2. $p_{\text {rms }}^{\prime}$ remains nearly unchanged for $\phi \leqslant 0.61$. In contrast, $E_{\text {trans }}$ increases as $\phi$ decreases from 0.61 to 0.49 , close to LBO, which shows that the translation error clearly captures the significant change in combustion close to LBO. 
combustor was considered, the dynamic behavior of the pressure fluctuations observed in this work becomes complex as the combustion state approaches LBO. $p_{\text {rms }}^{\prime}$ gradually decreases with decreasing $\phi$ below $\phi=0.70$. It drastically decreases when the intermittent pressure fluctuations occur but remains nearly unchanged for $\phi \leqslant 0.61$. This means that the standard deviation of the pressure fluctuations cannot capture the change in the complex combustion state close to LBO, which indicates that it is difficult to determine an appropriate threshold of $p_{\text {rms }}^{\prime}$ for detecting LBO. $E_{\text {trans }}$ drastically increases with the appearance of the intermittency in $p^{\prime}$, and monotonically increases with decreasing $\phi$ down to 0.49 in close proximity to LBO. The important point to note in Fig. 5 is that the translation error captures the significant change in the complex combustion state even under low equivalence ratio conditions close to LBO. This indicates that the translation error can be used to detect the precursor of LBO by setting a threshold. It is therefore of considerable interest to examine the possibility of controlling the combustion state using the translation error as an online detector. For this purpose, we control the combustion state so as to prevent $\mathrm{LBO}$ by continuing to capture the determinism governing the current temporal behavior of the pressure fluctuations.

An active control system using the power spectrum of temperature fluctuations has been adopted for preventing LBO during (I) power reduction and (II) transient processes [39]. In this work, we conduct closed-loop feedback control by adjusting the secondary fuel supply with the translation error used as the control variable to consider the above two processes. The time variation of the equivalence ratio $\phi$ with decreasing volume flow rate of the main fuel $Q_{\mathrm{CH} 4 \text {,main }}$ is shown in Fig. 6 as an example of power reduction. As shown by the dashed line in Fig. 6, only $Q_{\mathrm{CH} 4 \text {,main }}$ is decreased until LBO takes place. We attempt to control the combustion state by correlating the volume flow rate of the secondary fuel $Q_{\mathrm{CH} 4 \text {,second }}$ with the difference between $E_{\text {trans,thre }}$ and $E_{\text {trans }}$

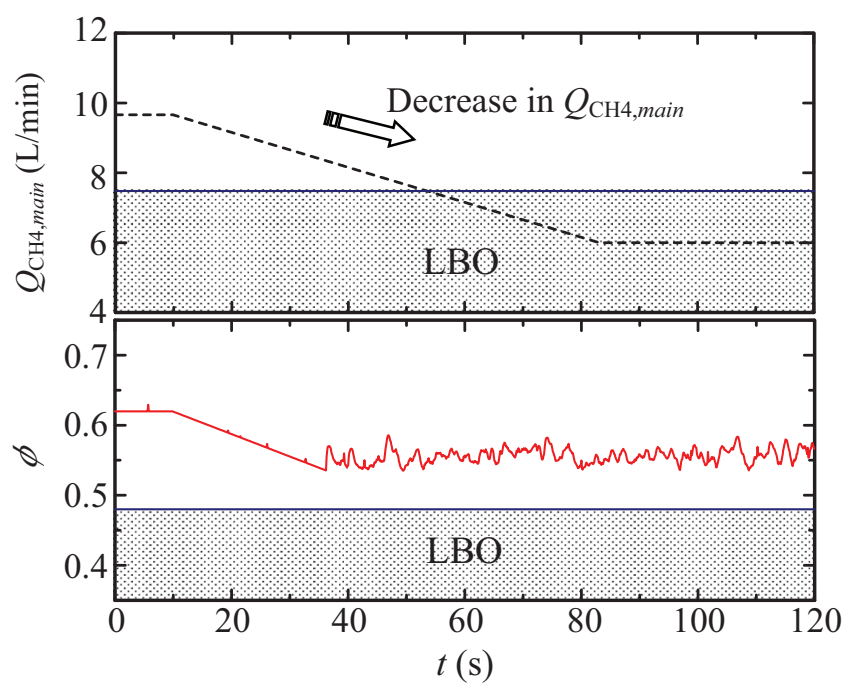

FIG. 6. (Color online) Time variation of equivalence ratio $\phi$ with decreasing volume flow rate of main fuel $Q_{\mathrm{CH} 4 \text {,main }}$. Lean blowout (LBO) is prevented even when $Q_{\mathrm{CH} 4 \text {,main }}$ is decreased to $6 \mathrm{~L} / \mathrm{min}$.

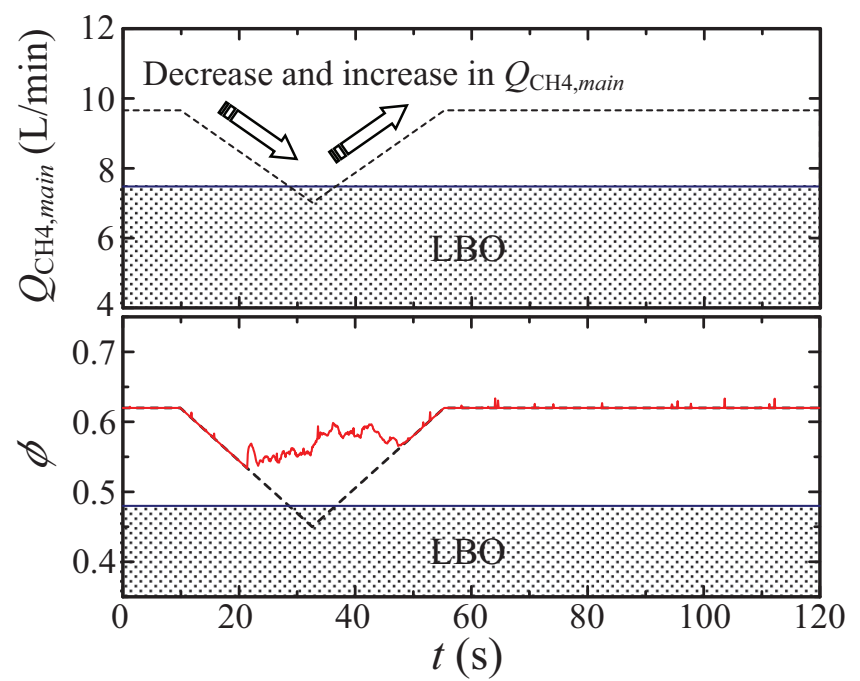

FIG. 7. (Color online) Time variation of equivalence ratio $\phi$ with decreasing and then increasing volume flow rate of main fuel $Q_{\mathrm{CH} 4 \text {,main. }}$. The prevention of LBO is achieved for the transient process as well as for the case of a power reduction.

$\left[Q_{\mathrm{CH} 4 \text {,second }}=\alpha\left(E_{\text {trans }}-E_{\text {trans,thre }}\right)\right]$. Here, $\alpha$ is a coefficient and $E_{\text {trans,thre }}$ is the threshold of $E_{\text {trans }}$ used for the control regulator. In this work, we set $E_{\text {trans,thre }}$ and $\alpha$ to 0.15 and 1.0, respectively. Our results clearly show that LBO is prevented even when $Q_{\mathrm{CH} 4 \text { main }}$ is decreased to $6 \mathrm{~L} / \mathrm{min}$. The time variation of the equivalence ratio $\phi$ with decreasing and then increasing the volume flow rate of the main fuel $Q_{\mathrm{CH} 4 \text {,main }}$ is shown in Fig. 7 as an example of a transient process. $Q_{\mathrm{CH} 4 \text {,main }}$ is decreased, leading to $\mathrm{LBO}$, but the addition of fuel from the secondary fuel supply system prevents LBO. After preventing LBO by increasing $Q_{\mathrm{CH} 4 \text {,main }}$, the second fuel supply system returns to the initial setting. These results indicate that without knowing the actual LBO limit, the online detection method using the translation error has considerable potential for preventing LBO in a premixed gas-turbine model combustor.

In reality, the noise inherently included in experimental systems inevitably affects the deterministic dynamics governing the temporal behavior of combustion instability. Although the translation error method in itself may be considered conventional in the fields of nonlinear science and physics, it can provide reliable estimates from a short time series and is robust to the noise contamination of obtained experimental data [31]. In this sense, the translation error involving the online update of a small amount of library data proposed in this work is applicable to combustion control, which will be worthwhile from the practical viewpoint of combustion systems. It is well recognized in the field of applied physics that plasma processing is an indispensable technology for the manufacture of semiconductors. A major drawback of plasma processing, however, is that the onset of anomalous discharges leads to serious problems such as damage to microdevices. With the aim of detecting the onset of anomalous discharges, the dynamical properties of acoustic emission by anomalous discharges in a plasma processing system has been investigated 
relatively recently [40]. It is considered that the online detection method proposed in this work has potential use for detecting anomalous discharges in the plasma processing system in Ref. [32]. In this sense, our work may provide useful guidelines for detecting and controlling dynamic behavior in a wide spectrum of complex nonlinear phenomena, including combustion phenomena. In this work, we have investigated the applicability of dynamical system theory as a useful detector to control the combustion state from a practical viewpoint. We need to examine whether and to what extent the online detection method we proposed is feasible for appropriately controlling the combustion state without the occurrence of LBO under a broad range of experimental conditions, as well as to reduce the response time of the current control system so that it can be applied to combustion instability with quite high frequencies. Our system enables us to control the combustion state up to $E_{\text {trans }}=0.20$, but the appropriate control of the combustion state close to the lean blow-out limit is difficult at present owing to the limitation of the current fuel flow control system used as an actuator. We demonstrated in this work that our method based on dynamical system theory is capable of preventing LBO in both (I) power reduction and (II) transient processes [39]. A possible application of our method in engineering is the operation control of gas-turbine engines, including the control of fuel flow-rate schedules. Typically, the frequency responses required for such control are less than $10 \mathrm{~Hz}$. The method of detecting instability proposed in this study should fulfill a key role in such control as it continuously provides updates of the status of monitored targets. The operation can be made more flexible by virtue of the updated information, eventually leading to stable and low-emission combustion. These issues will be focused on in our future work to enable the practical application of the online detection method based on orbital instability in the phase space for controlling the dynamic behavior of combustion instability.

\section{CONCLUSION}

We have proposed an online method of detecting combustion instability based on the concept of dynamical system theory, including investigation of the dynamic behavior of combustion instability. As an important case study relevant to combustion instability encountered in fundamental and practical combustion systems, we deal with combustion dynamics close to lean blowout (LBO) in a laboratory-scale premixed gasturbine model combustor. With decreasing equivalence ratio, irregular pressure fluctuations with small amplitudes suddenly appear in the relatively regular pressure fluctuations generated by thermoacoustic combustion oscillations, resulting in lowdimensional intermittent chaos. This is clearly shown using recurrence plots and the nonlinear forecasting method recently proposed by Gotoda and co-workers $[29,36]$. The translation error, which can be characterized by quantifying the degree of parallelism of trajectories in the phase space constructed from the short time series data of combustion-chamber pressure fluctuations, is used to detect the significant change in the combustion state under low equivalence ratio conditions. Our results show that the translation error has potential use as a control variable to prevent $\mathrm{LBO}$, which is worthwhile from the practical viewpoint of combustion systems.

\section{ACKNOWLEDGMENTS}

One of the authors (H.G.) was partially supported by a research grant from the Paloma Environmental Technology Foundation, the Adaptable \& Seamless Technology Transfer Program through Target-Driven R\&D from Japan Science and Technology Agency (JST), and a Grant-in-Aid for Young Scientists (A) from the Ministry of Education, Culture, Sports, Science, and Technology of Japan (MEXT). This work is based on many useful findings obtained by collaborative works with Prof. Takaya Miyano [20,29]. The authors would like to express their sincere thanks for his enormous contributions.
[1] S. Candel, Proc. Combust. Inst. 29, 1 (2002).

[2] Combustion Instabilities in Gas Turbine Engines: Operational Experience, Fundamental Mechanisms and Modeling, edited by T. C. Lieuwen and V. Yang, Progress in Astronautics and Aeronautics Vol. 210 (American Institute of Aeronautics and Astronautics, Reston, VA, 2005).

[3] K. Balasubramanian and R. I. Sujith, Phys. Fluids 20, 044103 (2008).

[4] L. Kabiraj, A. Saurabh, P. Wahi, and R. I. Sujith, Chaos 22, 023129 (2012).

[5] K. Kashinath, Ph.D thesis, University of Cambridge, 2013.

[6] T. Biwa, Y. Tashiro, U. Mizutani, M. Kozuka, and T. Yazaki, Phys. Rev. E 69, 066304 (2004).

[7] T. Biwa, Y. Tashiro, M. Ishigaki, Y. Ueda, and T. Yazaki, J. Appl. Phys. 101, 064914 (2007).

[8] T. Yoshida, T. Yazaki, H. Futaki, K. Hamaguchi, and T. Biwa, Appl. Phys. Lett. 95, 044101 (2009).

[9] T. Biwa, D. Hasegawa, and T. Yazaki, Appl. Phys. Lett. 97, 034102 (2010).
[10] H. Hatori, T. Biwa, and T. Yazaki, J. Appl. Phys. 111, 074905 (2012).

[11] G. Searby and D. Rochwerger, J. Fluid Mech. 231, 529 (1991).

[12] V. Bychkov, Phys. Fluids 11, 3168 (1998).

[13] X. Wu, M. Wang, P. Moin, and N. Peters, J. Fluid Mech. 497, 23 (2003).

[14] C. J. Lawn and W. Polifke, Combust. Sci. Technol. 176, 1359 (2004).

[15] W. Polifke and C. J. Lawn, Combust. Flame 151, 437 (2007).

[16] V'. Akkerman and C. K. Law, Phys. Fluids 25, 013602 (2013).

[17] J. Yáñez, M. Kuznetsov, and R. Redlinger, Combust. Flame 160, 2009 (2013).

[18] Y. Huang and V. Yang, Prog. Energy Combust. Sci 35, 293 (2009).

[19] T. C. Lieuwen, Unsteady Combustor Physics (Cambridge University Press, Cambridge, UK, 2012).

[20] H. Gotoda, H. Nikimoto, T. Miyano, and S. Tachibana, Chaos 21, 013124 (2011). 
[21] H. Gotoda, M. Amano, T. Miyano, T. Ikawa, K. Maki, and S. Tachibana, Chaos 22, 043128 (2012).

[22] Q. Zhang, S. J. Shanbhogue, and T. Lieuwen, J. Eng. Gas Turbines Power 132, 111502 (2010).

[23] H. Kantz and T. Schreiber, Nonlinear Time Series Analysis (Cambridge University Press, Cambridge, UK, 1997).

[24] C. S. Daw, J. F. Thomas, G. A. Richards, and L. L. Narayanaswami, Chaos 5, 662 (1995).

[25] T. Kaminski, M. Wendeker, K. Urbanowicz, and G. Litak, Chaos 14, 461 (2004).

[26] A. K. Sen, G. Litak, T. Kaminski, and M. Wendeker, Chaos 18, 033115 (2008).

[27] H. Gotoda, T. Miyano, and I. G. Shepherd, Phys. Rev. E 81, 026211 (2010).

[28] P. Grassberger and I. Procaccia, Phys. Rev. Lett. 50, 346 (1983).

[29] H. Gotoda, T. Ikawa, K. Maki, and T. Miyano, Chaos 22, 033106 (2012).

[30] A. C. Noble, G. B. King, N. M. Laurendeau, J. R. Gord, and S. Roy, Combust. Sci. Technol. 184, 293 (2012).
[31] R. Wayland, D. Bromley, D. Pickett, and A. Passamante, Phys. Rev. Lett. 70, 580 (1993).

[32] F. Takens, Dynamical Systems of Turbulence, Lecture Notes in Mathematics Vol. 898 (Springer, Berlin, 1981).

[33] L. Kabiraj and R. Sujith, J. Fluid Mech. 713, 376 (2012).

[34] S. Tachibana, J. Yamashita, L. Zimmer, K. Suzuki, and A. K. Hayashi, Proc. Combust. Inst. 32, 1795 (2009).

[35] J. Eckmann, S. O. Kamphorst, and D. Ruelle, Europhys. Lett. 4, 973 (1987).

[36] H. Gotoda, R. Takeuchi, Y. Okuno, and T. Miyano, J. Appl. Phys. 113, 124902 (2013).

[37] H. Gotoda, M. Pradas, and S. Kalliadasis (unpublished).

[38] M. Pradas, D. Tseluiko, S. Kalliadasis, D. T. Papageorgiou, and G. A. Pavliotis, Phys. Rev. Lett. 106, 060602 (2011).

[39] H. Li, X. Zhou, J. B. Jeffries, and R. K. Hanson, Proc. Combust. Inst. 31, 3215 (2007).

[40] T. Miyano, T. Moriya, H. Nagaike, N. Ikeuchi, and T. Matsumoto, J. Phys. D 41, 035209 (2008). 